\title{
ALMA sub-mm maser and dust distribution of VY Canis Majoris ${ }^{\star}$
}

A. M. S. Richards ${ }^{1}$, C. M. V. Impellizzeri ${ }^{2,4}$, E. M. Humphreys ${ }^{3}$, C. Vlahakis $^{4}$, W. Vlemmings ${ }^{5}$, A. Baudry ${ }^{6,7}$, E. De Beck ${ }^{5}$, L. Decin ${ }^{8}$, S. Etoka ${ }^{9}$, M. D. Gray ${ }^{1}$, G. M. Harper ${ }^{10}$, T. R. Hunter ${ }^{2}$, P. Kervella ${ }^{11,12,13}$, F. Kerschbaum ${ }^{14}$, I. McDonald ${ }^{1}$, G. Melnick ${ }^{15}$, S. Muller ${ }^{5}$, D. Neufeld ${ }^{16}$, E. O'Gorman ${ }^{5}$, S. Yu. Parfenov ${ }^{17}$, A. B. Peck ${ }^{2}$, H. Shinnaga ${ }^{18}$, A. M. Sobolev ${ }^{17}$, L. Testi ${ }^{3}$, L. Uscanga ${ }^{19}$, A. Wootten ${ }^{2}$, J. A. Yates ${ }^{20}$, and A. Zijlstra ${ }^{1}$

1 JBCA, School of Physics and Astronomy, Univ. of Manchester, UK e-mail: amsr@jb.man.ac.uk

2 NRAO, 520 Edgemont Road, Charlottesville, VA22903, USA

3 ESO Karl-Schwarzschild-Str. 2, 85748 Garching, Germany

4 Joint ALMA Observatory/European Southern Observatory, 3107 Alonso de Cordova, Vitacura, Santiago, Chile

5 Department of Earth and Space Sciences, Chalmers University of Technology, Onsala Space Observatory, 43992 Onsala, Sweden

${ }^{6}$ Univ. Bordeaux, LAB, UMR 5804, 33270 Floirac, France

7 CNRS, LAB, UMR 5804, 33270 Floirac, France

8 Instituut voor Sterrenkunde, Katholieke Universiteit Leuven, Celestijnenlaan 200D, 3001 Leuven, Belgium

9 Hamburger Sternwarte, Univ. of Hamburg, Gojenbergsweg 112, 21029 Hamburg, Hamburg, Germany

10 School of Physics, Trinity College Dublin, 2 Dublin, Ireland

11 LESIA, Observatoire de Paris, CNRS, UPMC, Université Paris-Diderot, PSL, 5 place Jules Janssen, 92195 Meudon, France

12 UMI-FCA, CNRS/INSU (UMI 3386), France

13 Dept. de Astronomía, Universidad de Chile, Casilla 36-D Santiago, Chile

14 Dept. of Astrophysics, Univ. of Vienna, Türkenschanzstraße 17, 1180 Vienna, Austria

15 Harvard-Smithsonian Center for Astrophysics, 60 Garden Street, MS 66, Cambridge, MA 02138, USA

16 Dept. of Physics \& Astronomy, Johns Hopkins Univ., 3400 North Charles Street, Baltimore, MD 21218, USA

17 Ural Federal University, Ekaterinburg, Russia

18 NAOJ, 2-21-1 Osawa, Mitaka, 181-8588 Tokyo, Japan

19 IAASARS, National Observatory of Athens, 15236 Athens, Greece

20 Dept. of Physics and Astronomy, University College London, WC1E 6BT, UK

Received 18 September 2014 / Accepted 16 October 2014

\section{ABSTRACT}

\begin{abstract}
Aims. Cool, evolved stars have copious, enriched winds. Observations have so far not fully constrained models for the shaping and acceleration of these winds. We need to understand the dynamics better, from the pulsating stellar surface to $\sim 10$ stellar radii, where radiation pressure on dust is fully effective. Asymmetric nebulae around some red supergiants imply the action of additional forces. Methods. We retrieved ALMA Science Verification data providing images of sub-mm line and continuum emission from VY CMa. This enables us to locate water masers with milli-arcsec accuracy and to resolve the dusty continuum.

Results. The 658, 321, and $325 \mathrm{GHz}$ masers lie in irregular, thick shells at increasing distances from the centre of expansion. For the first time this is confirmed as the stellar position, coinciding with a compact peak offset to the NW of the brightest continuum emission. The maser shells overlap but avoid each other on scales of up to 10 au. Their distribution is broadly consistent with excitation models but the conditions and kinematics are complicated by wind collisions, clumping, and asymmetries.
\end{abstract}

Key words. stars: individual: VY CMa - supergiants - stars: mass-loss - masers

\section{Introduction}

Massive stars have a profound impact on their surroundings via their material and energy output. Observations support the importance of radiation pressure on dust in driving the stellar wind, as reviewed by Habing (1996) and confirmed for red supergiants (RSG) by Mauron \& Josselin (2011). There are a variety of models for mass transport from the stellar surface to the dust formation zone at $5-10$ stellar radii $\left(R_{\star}\right)$, based on combinations of convection (Chiavassa et al. 2011), wind levitation by pulsation and dust formation (Bowen 1988; Ireland \& Scholz 2006), for example, including scattering as well as absorption (Bladh et al. 2013). Acoustic and magnetic forces were analysed

\footnotetext{
* Appendices are available in electronic form at http://www . aanda.org
}

by Hartmann \& MacGregor (1980). However, observations do not yet confirm any of these as the dominant force. Oxygen-rich grain formation models have difficulty in explaining dust-driven winds (Woitke 2006), which can possibly be solved by the detection of large dust grains at a few $R_{\star}$ (Norris et al. 2012) and the complexity of the gas and dust distribution close to the photosphere (Wittkowski et al. 2007). Moreover, most investigations have focused on low-mass stars, which have a different internal structure, and despite their high mass-loss rates, RSG have irregular, often shallow periods ${ }^{1}$.

We investigated mass loss from VY CMa, one of the largest RSG, progenitor mass $\left.\sim 25 M_{\odot}, R_{\star}\right) 5.7$ mas at $2 \mu \mathrm{m}$ (Wittkowski et al. 2012), at $1.2 \pm 0.1 \mathrm{kpc}$, Choi et al. 2008;

1 http://cdsarc.u-strasbg.fr/cgi-bin/afoevList?cma/vy 
Zhang et al. 2012). It has had a high and variable mass-loss rate, $0.5-1 \times 10^{-4} M_{\odot} \mathrm{yr}^{-1}$ in its recent past (Decin et al. 2006), up to $3 \times 10^{-3} M_{\odot} \mathrm{yr}^{-1}$ (Humphreys et al. 2007). This provides the richest-known O-rich circumstellar envelope (CSE) chemistry, as seen at sub-mm wavelengths by Herschel (Alcolea et al. 2013), for instance, and imaged at $\sim 1^{\prime \prime}$ resolution using the Submillimeter Array (SMA, Kamiński et al. 2013).

VY CMa has a highly asymmetric nebula that extends over a few arcsec and is shaped like a lopsided heart, irregular and clumpy on all scales (Humphreys et al. 2007). Very Large Array (VLA) and SMA observations at $8.4-355 \mathrm{GHz}$ show an unresolved central ellipse, dominated by emission from dust, (e.g. Lipscy et al. 2005; Kamiński et al. 2013). Strong OH, SiO, and $22 \mathrm{GHz} \mathrm{H}_{2} \mathrm{O}$ masers have been imaged by many authors but, hitherto, there has been no astrometric confirmation that the star lies at the centre of expansion. The $22 \mathrm{GHz} \mathrm{H}_{2} \mathrm{O}$ masers are located in a thick shell of radii 75-440 mas, with Doppler and proper motions dominated by accelerating outflow (Richards et al. 1998). Their maximum expansion velocity is $35.5 \mathrm{~km} \mathrm{~s}^{-1}$ relative to the stellar velocity $V_{\star}$ of $22 \mathrm{~km} \mathrm{~s}^{-1}$ (all velocities are with respect to the local standard of rest, LSR).

Models (Gray 2012 and references therein; Daniel \& Cernicharo 2013) predict that the $321.22564 \mathrm{GHz} J_{K \mathrm{a}, K \mathrm{c}} 10_{2,9}-$ $9_{3,6}$, and possibly the $325.15292 \mathrm{GHz} 5_{1,5}-4_{2,2} \mathrm{H}_{2} \mathrm{O}$ maser lines can emanate from conditions found at both sides of the dust formation zone. Maser emission at $321 \mathrm{GHz}$ needs hotter gas than at $22 \mathrm{GHz}$, whilst lower temperatures and number densities favour $325 \mathrm{GHz}$. This has been confirmed by imaging in Cepheus A (Patel et al. 2007), but only the $22 \mathrm{GHz}$ transition has ever been resolved in a CSE. The $658.00655 \mathrm{GHz} v_{2}=1,1_{1,0}-1_{0,1} \mathrm{GHz}$ maser is expected to occur very close to the star under conditions similar to $\mathrm{SiO}$ masers (Hunter et al. 2007). More details of these transitions are given in Table A.1. We present ALMA observations that test these predictions and, for the first time, resolve sub-mm masers, thermal lines, and continuum.

\section{Data acquisition and reduction}

We obtained public ALMA Science Verification data for VY CMa observed on 2013 16-19 August using 16-20 12 m antennas on baselines from $0.014-2.7 \mathrm{~km}$. Three scheduling blocks (SB), covering each of the maser lines, are referred to as the $321 \mathrm{GHz}, 325 \mathrm{GHz}$ and $658 \mathrm{GHz}$ SBs. The velocity resolution after Hanning smoothing is $0.45 \mathrm{~km} \mathrm{~s}^{-1}$ at 321 and $658 \mathrm{GHz}$, and $0.9 \mathrm{~km} \mathrm{~s}^{-1}$ at $325 \mathrm{GHz}$. More details of observations and data reduction are given in Appendix B. The fully calibrated line-free channels were imaged using a synthesized beam of $\left(0.22 \times 0 .{ }^{\prime} 13\right)$ at 321 and $325 \mathrm{GHz}$, and $\left(00^{\prime} 11 \times 00^{\prime} 06\right)$ at $658 \mathrm{GHz}$. After subtracting the continuum, the masers were imaged using beam sizes of $\left(0{ }^{\prime} 18 \times 00^{\prime} 09\right)$ and $\left(0{ }^{\prime} 088 \times 00^{\prime} 044\right)$ at $321 / 325$ and $658 \mathrm{GHz}$. We measured the positions of the masers and continuum peaks by fitting Gaussian components using the AIPS task SAD, see Appendix B.

\section{VY CMa continuum and maser morphology}

Before self-calibration, the positions measured by Gaussian fits to the 321 and $325 \mathrm{GHz}$ peaks wandered by up to 35 mas, but at $658 \mathrm{GHz}$ there were offsets of up to 100 mas, mainly due to differences between atmospheric conditions towards VY CMa and the phase reference source (see Appendix B). The $321 \mathrm{GHz}$ data, with the best atmospheric transmission and sensitivity, were used for astrometry and for continuum analysis around this frequency. The continuum at all frequencies has a similar $\mathrm{J}$ shape, with a

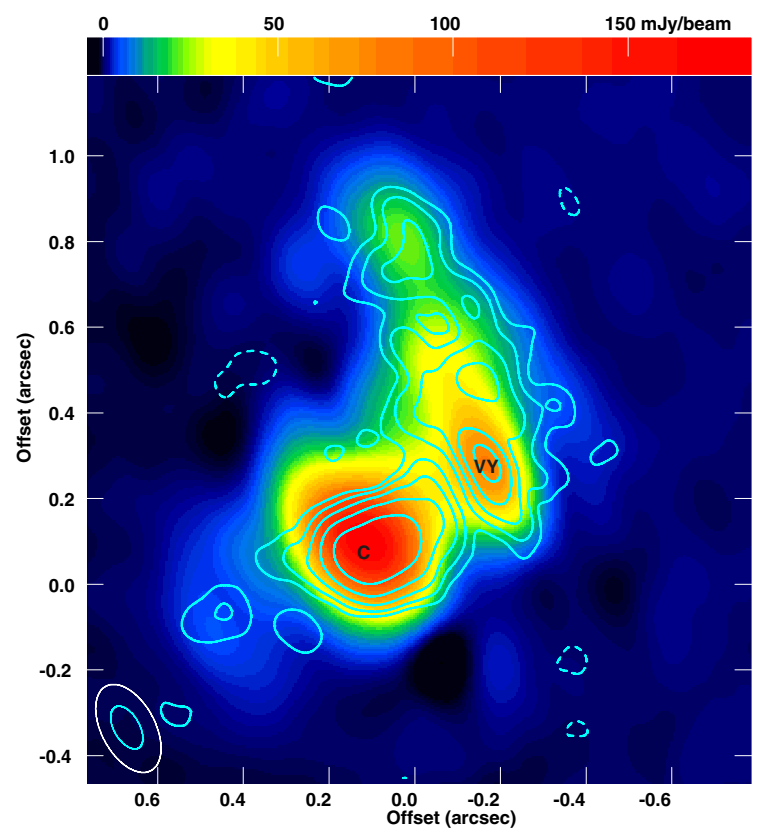

Fig. 1. Continuum emission: $321 \mathrm{GHz}$ colour scale, $658 \mathrm{GHz}$ contours at $(-1,1,2,4,8,16) \times 10 \mathrm{mJy}^{\text {beam }}{ }^{-1}$. Synthesized beams shown at lower left for $321 \mathrm{GHz}$ (white), $658 \mathrm{GHz}$ (blue). (0, 0) at RA 0722 58.33454 Dec -254603.3275 (J2000). C marks the continuum peak. $\mathrm{VY}$ is identified as the star, at the centre of the water maser expansion.

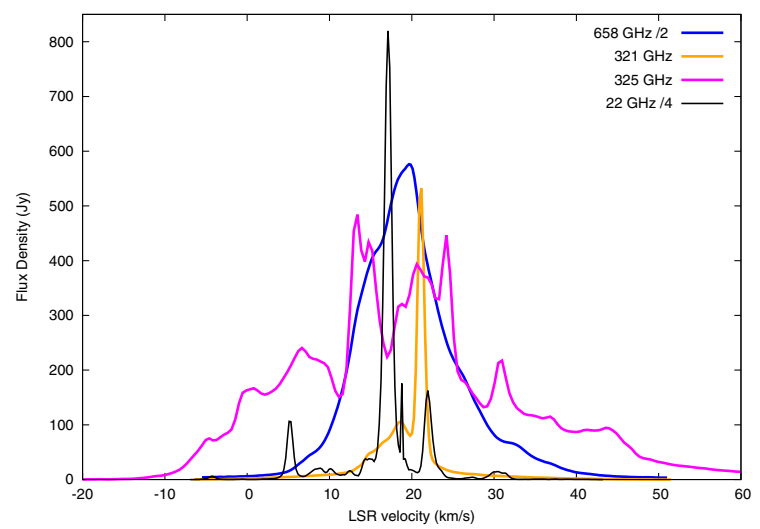

Fig. 2. Integrated water maser spectra, derived from the interferometric image cubes, measured in square boxes of width $0.75,1^{\prime \prime} .0$ and 0.4 at 321,325 , and $658 \mathrm{GHz}$, centred on VY. The $658 \mathrm{GHz}$ spectrum is scaled by 0.5 and the $22 \mathrm{GHz}$ spectrum by 0.25 .

bright extended peak C, a secondary, compact peak VY, and several other peaks. Shifts of $(-2,0)$ and $(-87,27)$ mas were applied at 325 and $658 \mathrm{GHz}$, respectively, to align VY with its $321 \mathrm{GHz}$ position, which brought the other bright features into good positional agreement (Fig. 1). The maximum detectable angular extent in RA and Dec is $1^{\prime \prime} .2 \times 1$.' 6 above the $3 \sigma_{\text {rms }}$ contour at $321 \mathrm{GHz}$. The $321 \mathrm{GHz}$ position of VY is RA 072258.3226 Dec -25 4603.043 (J2000), with 35 mas uncertainty dominated by errors in transferring phase corrections from the reference source. VY is $328 \pm 1$ mas from $\mathrm{C}$ at PA $-33^{\circ}$. Using a matching beam size $160 \times 64 \mathrm{mas}^{2}, \mathrm{C}$ and VY had peak flux densities of 133.9 and $71.7\left(\sigma_{\text {rms }} 0.9\right)$ mJy beam $^{-1}$ at $321 \mathrm{GHz}$ and of 474 and $296\left(\sigma_{\text {rms }} 4\right)$ mJy beam $^{-1}$ at $658 \mathrm{GHz}$. The continuum emission is analysed further by O'Gorman et al. (2014).

The total velocity extents of the $\mathrm{H}_{2} \mathrm{O}$ lines are $(-11.9$ to 49.2), (-16.8 to 75.1) and (-3.0 to 67.7$) \mathrm{km} \mathrm{s}^{-1}$ at 321,325 , and $658 \mathrm{GHz}$. Figure 2 shows that the $325 \mathrm{GHz}$ maser spectrum has 

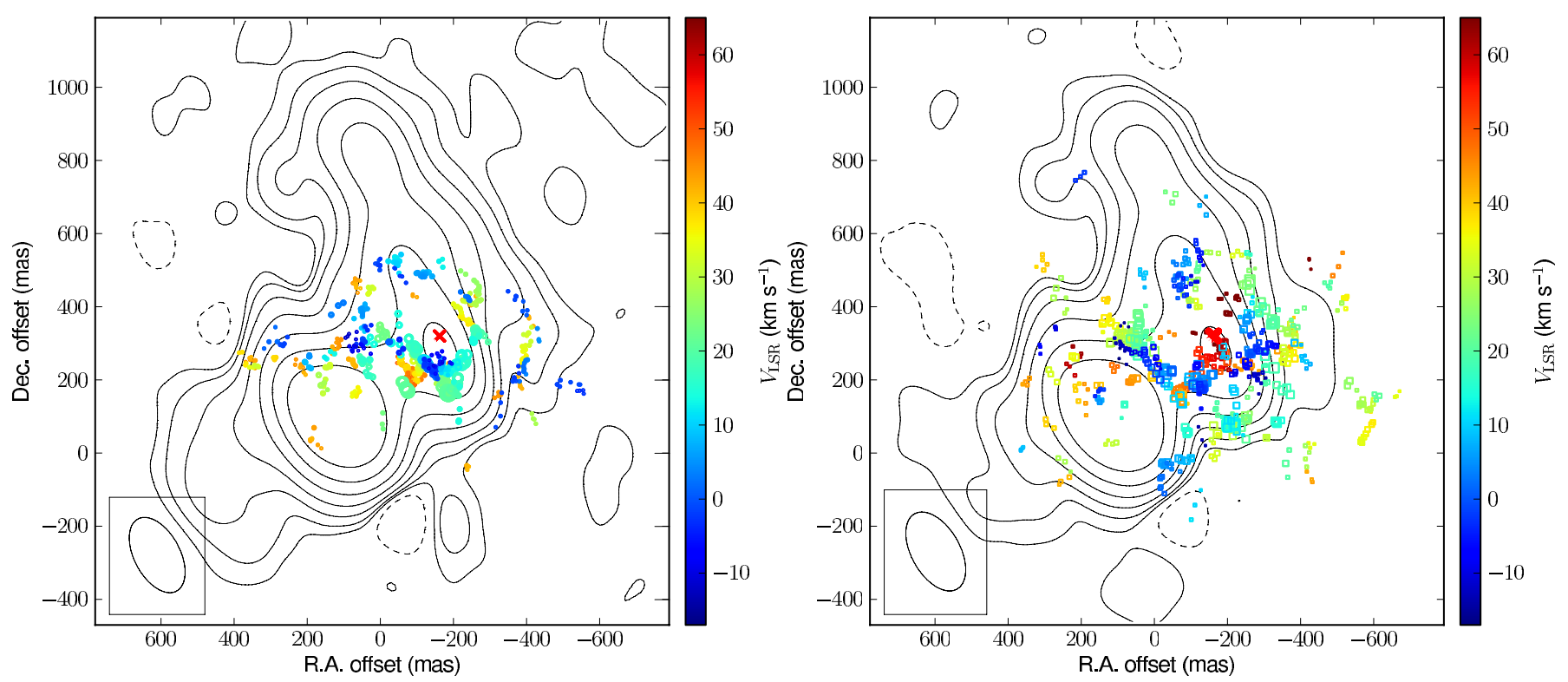

Fig. 3. $321 \mathrm{GHz}$ (left) and $325 \mathrm{GHz}$ (right) maser positions over continuum contours, lowest levels at $(-1,1)$ and $(-2) \mathrm{mJy}$ beam ${ }^{-1}$ at 321 and

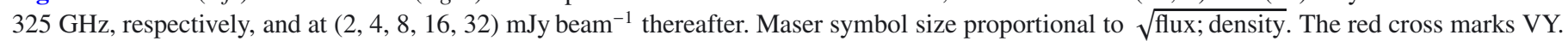

the largest velocity extent and shows multiple strong peaks (similar to $22 \mathrm{GHz}$ ), whilst the 321 and $658 \mathrm{GHz}$ spectra are dominated by single peaks, close to $V_{\star}$ at $321 \mathrm{GHz}$, but $2 \mathrm{~km} \mathrm{~s}^{-1}$ more blue-shifted at $658 \mathrm{GHz}$. Both these lines have a blue shoulder. The 325 and $658 \mathrm{GHz}$ masers have a long red tail.

We estimated the brightness temperature $T_{\mathrm{b}}$ of each spatial component from its measured flux density $S$ (Appendix B) and the beam size, that is, $T_{\mathrm{b}} \geq 756 \mathrm{~S}$ at 321 and $325 \mathrm{GHz}$, and $T_{\mathrm{b}} \geq 3162 \mathrm{~S}$ at $658 \mathrm{GHz}$. These give ranges of $\left(11-3.2 \times 10^{5}\right) \mathrm{K}$, $\left(46-2.0 \times 10^{5}\right) \mathrm{K}$, and $\left(444-28 \times 10^{5}\right) \mathrm{K}$ at 321, 325, and $658 \mathrm{GHz}$. These values are lower limits since maser component sizes are probably smaller than the beam; $\leq 5$ mas components would increase $T_{\mathrm{b}} \gg 1000 \mathrm{~K}$ in all cases, for instance. However, some of the faintest emission, especially at extreme velocities, may be genuinely extended, thermal emission from $\mathrm{H}_{2} \mathrm{O}$ or other species.

Figures 3 and 4 show that the masers are clumped into spatially close groups at similar velocities. Much of the 321 and $658 \mathrm{GHz}$ emission is concentrated in bright streamers with clear velocity gradients, for example in Fig. 4 (insert), where the long arc traces a continuum "valley" between C and VY. The $325 \mathrm{GHz}$ masers are more evenly distributed. There is more extended emission to the east of VY in all lines and brighter sub-mm masers to the south. The $321 \mathrm{GHz}$ masers form a ring around VY; the other lines have more blue- or red-shifted emission along the line of sight to VY (Figs. 3-5). We estimated the centre of expansion of each line as in Richards et al. (2012), by maximising the separation between masers at close to $V_{\star}$, giving positions very close to VY. Figure 6 shows the angular separation of each component from VY, which appears to be located in a maser-free sphere. Figures 6 and 5 show that the 658 , 321 , and $325 \mathrm{GHz}$ maser inner rims are at successively larger distances from the star, whilst the outer rims are at $\sim 200,500$, and 600 mas.

\section{Discussion}

VY coincides with the maser centre of expansion and is almost certainly the location of the star. This was suspected (Muller et al. 2007; Kamiński et al. 2013), but never before resolved as

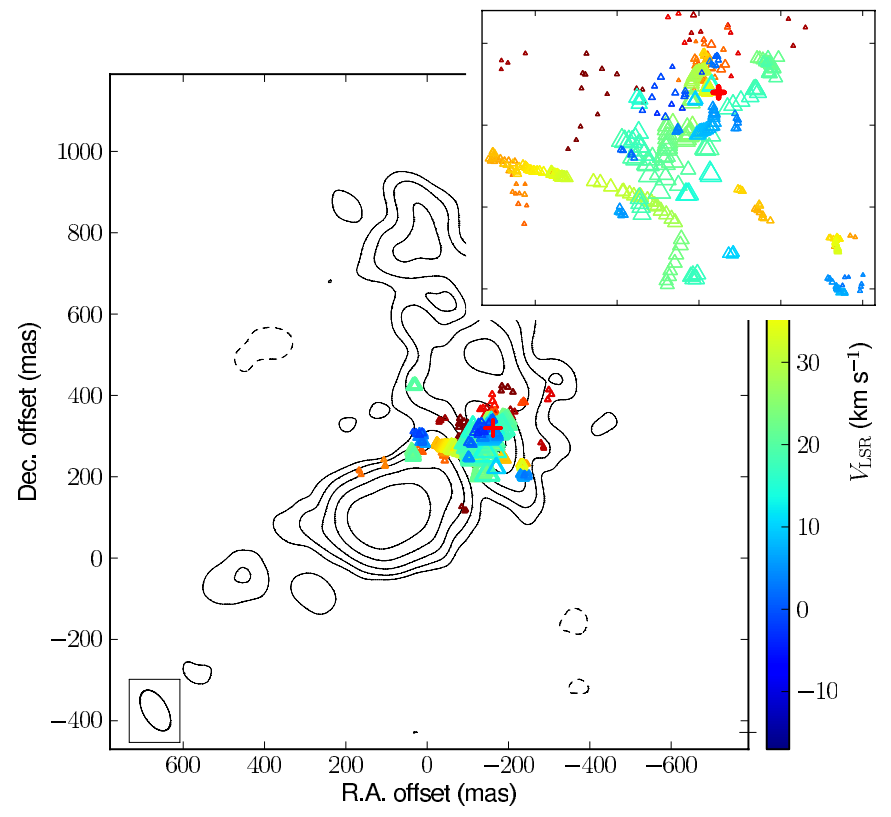

Fig. 4. $658 \mathrm{GHz} \mathrm{H}_{2} \mathrm{O}$ maser positions overlying continuum contours as in Fig. 1. Maser symbols and velocity scale as in Fig. 3. The inset shows the region with bottom left and top right at $(-20,190)$ and $(-255$, 370) mas.

a distinct continuum peak. Our position (Sect. 3) agrees within the uncertainties with the centre of expansion of $\mathrm{SiO}$ and $22 \mathrm{GHz}$ $\mathrm{H}_{2} \mathrm{O}$ masers (Zhang et al. 2012; Choi et al. 2008).

Exponential maser amplification exaggerates small differences in conditions, so single-epoch data are interpreted with caution. The $658 \mathrm{GHz}$ masers have a complex distribution close to the star, but mostly outside the typical $\mathrm{SiO}$ outer rim, taken from Richter et al. (2013), as shown in Fig. 6 (although Shinnaga et al. (2004) found a more extended bipolar outflow). The asymmetric emission at large angular separations and expansion velocities (Fig. 6) is inconsistent with a simple velocityradius relationship. The inner $658 \mathrm{GHz}$ masers arise in the 


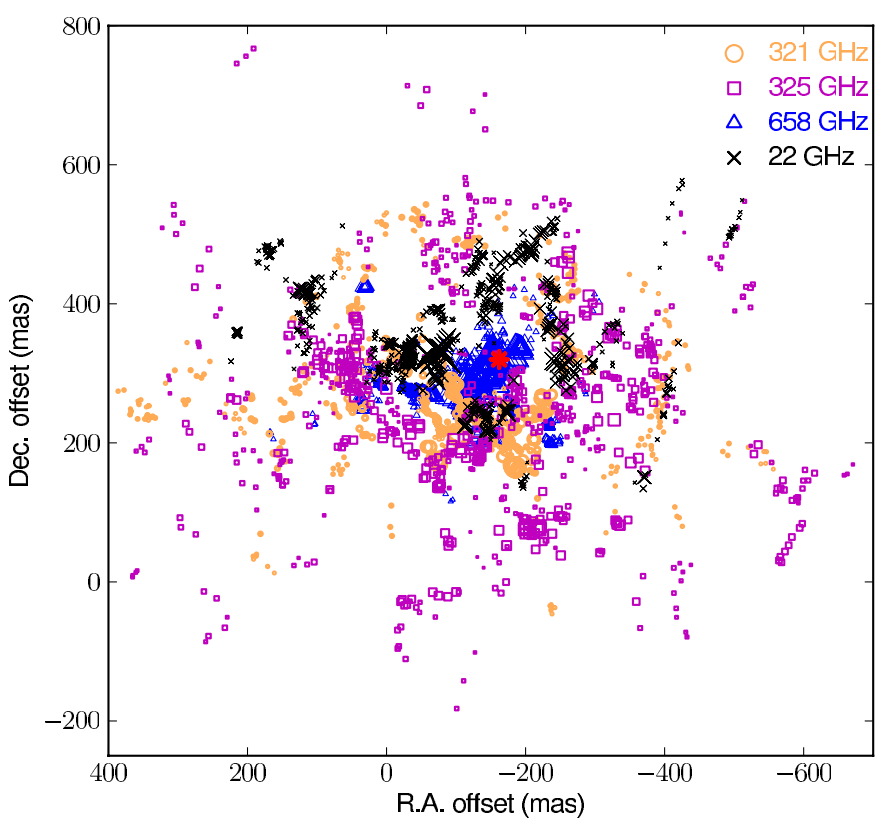

Fig. 5. Relative positions of all imaged maser components.

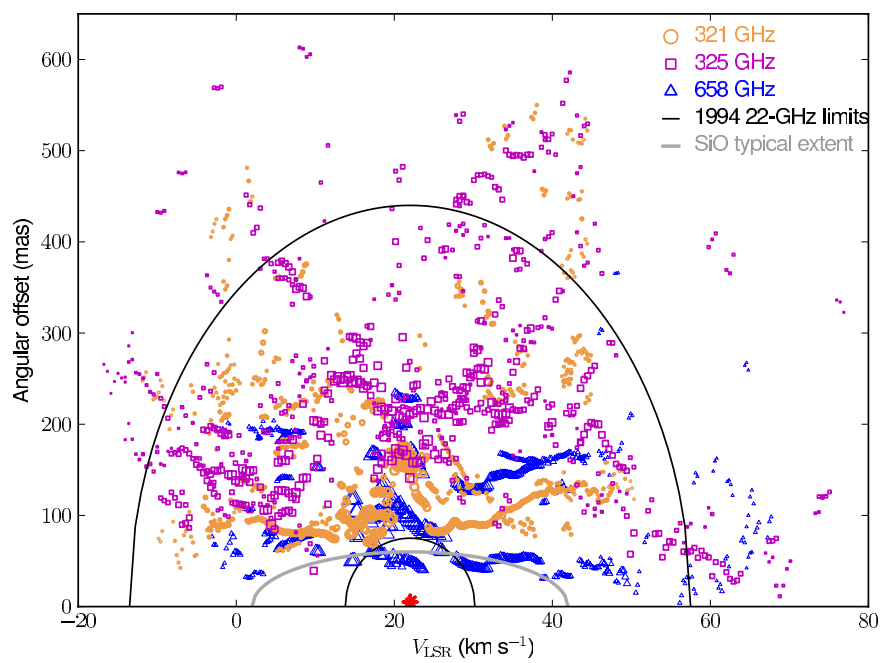

Fig. 6. Symbols mark sub-mm $\mathrm{H}_{2} \mathrm{O}$ maser component angular separations from VY as a function of $V_{\mathrm{LSR}}$. The black and grey lines mark the inner and outer limits of $22 \mathrm{GHz} \mathrm{H}_{2} \mathrm{O}$ masers and the outer rim of $J=1-0$ and $J=2-1 \mathrm{SiO}$ masers.

pulsation-dominated region, but straddle the dust formation zone identified by Decin et al. (2006) and indicated by the $22 \mathrm{GHz}$ inner radius.

The patchy ring of bright 321 and $325 \mathrm{GHz}$ masers within $\sim 10 \mathrm{~km} \mathrm{~s}^{-1}$ of $V_{\star}$ (Fig. 3) is typical of tangential beaming from a radially accelerating shell. The $321 \mathrm{GHz}$ masers are undetectable towards the star, suggesting that they trace particularly strongly accelerated gas. The red- and blue-shifted emission at large angular separations suggests additional, complex motions. The brightest $325 \mathrm{GHz}$ masers occur at velocities close to $V_{\star}$, seen in Fig. 7, but asymmetries are seen in the south and east offsets of the moderately blue- and red-shifted peaks. Figures 3 and 4 suggest that the masers, especially at $658 \mathrm{GHz}$, trace shocks where the stellar wind encounters continuum peak $\mathrm{C}$. Elongated, bright maser features characteristic of shocks, seen at up to $\sim 150$ mas from the star, could be associated with dust

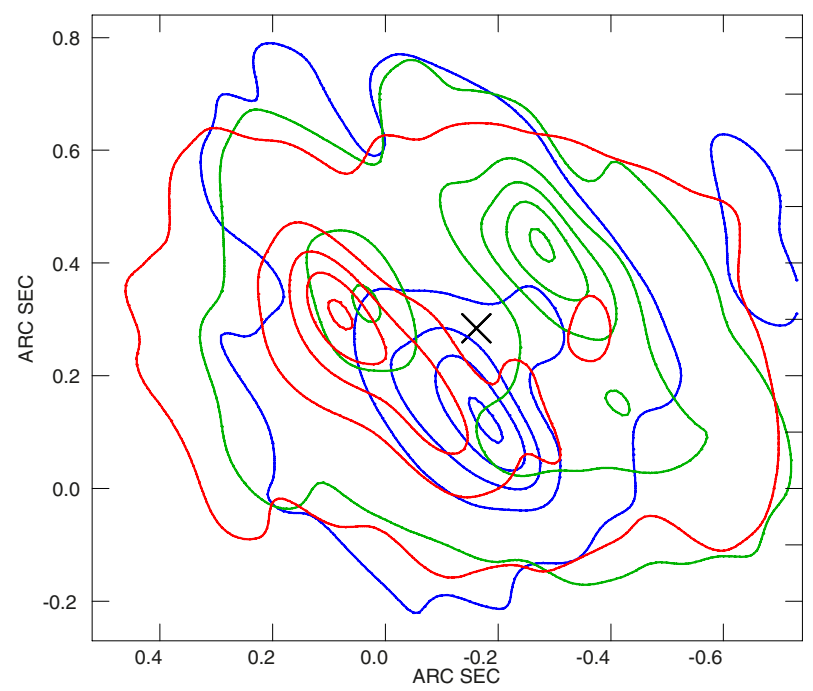

Fig. 7. $325 \mathrm{GHz}$ masers, summed over $<17,17-27$, and $>27 \mathrm{~km} \mathrm{~s}^{-1}$, shown in blue, green, and red. Contour levels start at $30 \mathrm{Jy}$; higher levels are at $24 \%, 48 \%, 72 \%$, and $96 \%$ of the peak, where the peak is 2184, 1018, and $903 \mathrm{Jy}$ for the red, green, and blue ranges. These levels have been chosen to emphasise the brighter emission and indicate the total extent at a uniform sensitivity limit (to $1-3 \%$ peak), omitting fine details. The cross marks VY.
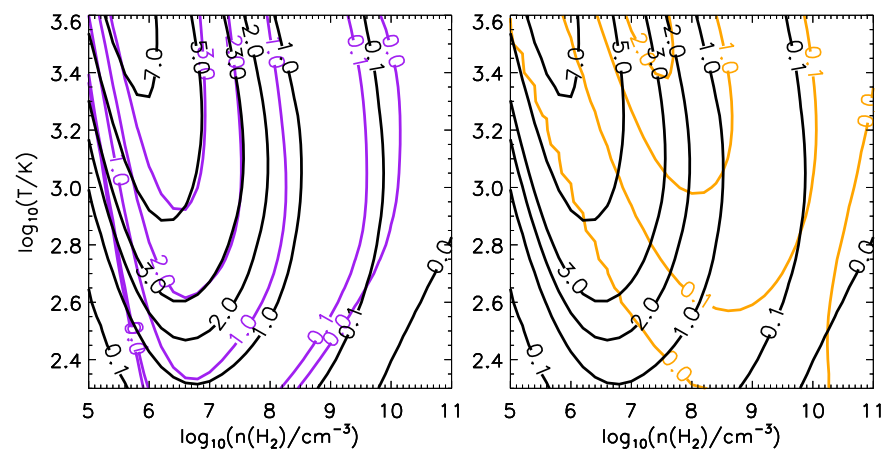

Fig. 8. Maser (negative) optical depths for $22 \mathrm{GHz}$ (black, both panels), $325 \mathrm{GHz}$ masers (magenta, left panel) and $321 \mathrm{GHz}$ (orange, right panel). Amplification occurs if this exceeds zero.

formation (Bladh et al. 2013) or wind collisions arising from inhomogenous mass loss/speeds (Zijlstra et al. 2001).

We grouped the maser components into features and attempted to cross-match the clumps of different transitions, but found no significant associations, so co-propagation of these sub-mm masers seems unlikely. A comparison with $22 \mathrm{GHz}$ was not made because of the difference in epochs; around similar evolved stars these are concentrated in clumps with a filling factor $\lesssim 1 \%$, containing 30-90\% of the total mass loss in this region (Richards et al. 2012), embedded in less dense gas.

The lines studied arise from distinct, although overlapping zones, and are segregated on scales of a few tens of au. All are thought to be collisionally pumped (Yates et al. 1997). Figure 8 compares the physical conditions required for masing of the three vibrational ground-state lines using an excitation model similar to that described by Neufeld \& Melnick (1991) updated as detailed in Appendix C. The conditions in the VY CMa CSE lie around the locus from top right (near the star) to bottom left, but with a variation of up to $\times 70$ in number density and $\times 0.7$ in temperature in denser clumps or compressed material. The contours mark the negative optical depths in the direction of the velocity gradient for unsaturated masers; the optical depths in 
other directions may greatly exceed the values shown here (optical depths of $22 \mathrm{GHz}$ masers have been estimated at up to $\sim 13$ in RSG S Per, Richards et al. 2011). The $321 \mathrm{GHz}$ gain drops rapidly below $1000 \mathrm{~K}$, whilst the 22 and $325 \mathrm{GHz}$ masers extend to cooler temperatures. The $325 \mathrm{GHz}$ maser is collisionally quenched at lower densities than the $22 \mathrm{GHz}$ and $321 \mathrm{GHz}$ transitions.

The models of Humphreys et al. (2001) predicted a comparable radial range for 22 and $325 \mathrm{GHz}$ masers around lowmass stars, the latter being favoured in cooler, more rarefied conditions, possibly in a two-phase medium. These predictions agree well with the observed inner radii at successively higher distances from the star at 321, 22, and $325 \mathrm{GHz}$, if the inner radii of 321 and $325 \mathrm{GHz}$ masers are determined by conditions in the inter-clump gas surrounding the $22 \mathrm{GHz}$ clouds. Not yet published models (Gray) require temperatures $>1500 \mathrm{~K}$ and $\mathrm{H}_{2}$ number densities $\sim 6 \times 10^{9} \mathrm{~cm}^{-3}$ for $658 \mathrm{GHz}$ masers, likely to be found in the inner parts of its observed distribution, but the more extended emission is surprising. The excitation of $321 \mathrm{GHz}$ masers at 500 mas $(600 \mathrm{au})$ from the star is also puzzling since typical temperatures of $250 \mathrm{~K}$ (Decin et al. 2006) are too low, so local heating might be important.

\section{Summary}

These ALMA observations have identified the centre of expansion of high-excitation masers with a distinct continuum peak, VY CMa itself, 360 au NW of the brightest sub-mm dust emission. The 658, 321, and $325 \mathrm{GHz}$ masers are found at increasing distances from the star as predicted, but reach unexpectedly large separations. The high-excitation 658 and possibly $321 \mathrm{GHz}$ masers cross the dust formation zone and some emission appears to emanate from shocked regions surrounding the star and tracing the limbs of $\mathrm{C}$; interaction with the uniquely complex dust distribution is undoubtedly significant. The different transitions form clumps that do not overlap even when found at similar separations from VY. The velocities are generally consistent with expansion but deviate drastically and irregularly from spherical symmetry. We will compare the maser kinematics with models of a flared disc plus bipolar outflow (Decin et al. 2006; Muller et al. 2007) and ejection along less symmetric arcs as described by Humphreys et al. (2007). These observations provide the first opportunity to test sub-mm maser models rigorously, to be followed-up in future papers along with an analysis of the kinematics, thermal lines, and dust.

Acknowledgements. This paper makes use of ALMA data: ADS/JAO.ALMA \#2011.0.00011.SV. ALMA is a partnership of ESO (representing its member states), NSF (USA) and NINS (Japan), together with NRC (Canada) and NSC and ASIAA (Taiwan), in cooperation with the Republic of Chile. The Joint
ALMA Observatory is operated by ESO, AUI/NRAO and NAOJ. We thank the anonymous referee for thoughtful comments that have greatly improved the clarity of this paper

\section{References}

Alcolea, J., Bujarrabal, V., Planesas, P., et al. 2013, A\&A, 559, A93

Bladh, S., Höfner, S., Nowotny, W., Aringer, B., \& Eriksson, K. 2013, A\&A 553, A20

Bowen, G. H. 1988, ApJ, 329, 299

Chiavassa, A., Freytag, B., Masseron, T., \& Plez, B. 2011, A\&A, 535, A22

Cheung, A. C., Rank, D. M., Townes, C. H., Thornton, D. D., \& Welch, W. J. 1969, Nature, 221, 626

Choi, Y. K., Hirota, T., Honma, M., et al. 2008, PASJ, 60, 1007

Condon, J. J., Cotton, W. D., Greisen, E. W., et al. 1998, AJ, 115, 1693

Daniel, F., \& Cernicharo, J. 2013, A\&A, 553, A70

Daniel, F., Dubernet, M.-L., \& Grosjean, A. 2011, A\&A, 536, A76

Decin, L., Hony, S., de Koter, A., et al. 2006, A\&A, 456, 549

Fu, R. R., Moullet, A., Patel, N. A., et al. 2012, ApJ, 746, 42

Gray, M. 2012, Maser Sources in Astrophysics (Cambridge University Press)

Habing, H. J. 1996, A\&ARv, 7, 97

Hartmann, L., \& MacGregor, K. B. 1980, ApJ, 242, 260

Humphreys, E. M. L., Yates, J. A., Gray, M. D., Field, D., \& Bowen, G. H. 2001 A\&A, 379, 501

Humphreys, R. M., Helton, L. A., \& Jones, T. J. 2007, AJ, 133, 2716

Hunter, T. R., Young, K. H., Christensen, R. D., \& Gurwell, M. A. 2007, in IAU Symp. 242, eds. J. M. Chapman, \& W. A. Baan, 481

Ireland, M. J., \& Scholz, M. 2006, MNRAS, 367, 1585

Kamiński, T., Gottlieb, C. A., Young, K. H., Menten, K. M., \& Patel, N. A. 2013 , ApJS, 209, 38

Knapp, G. R., \& Woodhams, M. 1993, in Massive Stars: Their Lives in the Interstellar Medium, eds. J. P. Cassinelli, \& E. B. Churchwell, ASP Conf. Ser., 35, 199

Lipscy, S. J., Jura, M., \& Reid, M. J. 2005, ApJ, 626, 439

Mauron, N., \& Josselin, E. 2011, A\&A, 526, A156

Menten, K. M., \& Melnick, G. J. 1991, ApJ, 377, 647

Menten, K. M. \& Young, K. 1995, ApJ, 450, L67

Menten, K. M., Melnick, G. J., \& Phillips, T. G. 1991, ApJ, 350, L41

Muller, S., Dinh-V-Trung, Lim, J., et al. 2007, ApJ, 656, 1109

Neufeld, D. A. 2010, ApJ, 708, 635

Neufeld, D. A., \& Melnick, G. J. 1991, ApJ, 368, 215

Norris, B. R. M., Tuthill, P. G., Ireland, M. J., et al. 2012, Nature, 484, 220

O'Gorman, E., Vlemmings, W., Richards, A. M. S., et al. 2014, A\&A, submitted [arXiv: 1410.1622]

Patel, N. A., Curiel, S., Zhang, Q., et al. 2007, ApJ, 658, L55

Richards, A. M. S. 1997, Ph.D. Thesis, University of Manchester

Richards, A. M. S., Yates, J. A., \& Cohen, R. J. 1998, MNRAS, 299, 319

Richards, A. M. S., Elitzur, M., \& Yates, J. A. 2011, A\&A, 525, A56

Richards, A. M. S., Etoka, S., Gray, M. D., et al. 2012, A\&A, 546, A16

Richter, L., Kemball, A., \& Jonas, J. 2013, MNRAS, 436, 1708

Shinnaga, H., Moran, J. M., Young, K. H., \& Ho, P. T. P. 2004, ApJ, 616, L47

Wittkowski, M., Boboltz, D. A., Ohnaka, K., Driebe, T., \& Scholz, M. 2007, A\&A, 470, 191

Wittkowski, M., Hauschildt, P. H., Arroyo-Torres, B., \& Marcaide, J. M. 2012, A\&A, 540, L12

Woitke, P. 2006, A\&A, 460, L9

Yates, J. A., Field, D., \& Gray, M. D. 1997, MNRAS, 285, 383

Zhang, B., Reid, M. J., Menten, K. M., \& Zheng, X. W. 2012, ApJ, 744, 23

Zijlstra, A. A., te Lintel Hekkert, P., Chapman, J. M., et al. 2001, MNRAS, 322, 280 
Table A.1. $\mathrm{H}_{2} \mathrm{O}$ masers.

\begin{tabular}{cccccc}
\hline \hline $\begin{array}{c}\text { Frequency } \\
(\mathrm{GHz})\end{array}$ & $\begin{array}{c}\text { Transition } \\
\left(J_{K \mathrm{a}, K \mathrm{c}}\right)\end{array}$ & $v_{2}$ & $\begin{array}{r}E_{\mathrm{U}} \\
(\mathrm{K})\end{array}$ & $\begin{array}{c}\text { Spin } \\
(\text { level })\end{array}$ & $\begin{array}{c}\text { Discovery } \\
\text { (reference) }\end{array}$ \\
\hline 22.23508 & $6_{1,6}-5_{2,3}$ & 0 & 643 & $\mathrm{o}$ & $\mathrm{C69}$ \\
321.22564 & $10_{2,9}-9_{3,6}$ & 0 & 1862 & $\mathrm{o}$ & M90 \\
325.15292 & $5_{1,5}-4_{2,2}$ & 0 & 470 & $\mathrm{p}$ & M91 \\
658.00655 & $1_{1,0}-1_{0,1}$ & 1 & 2361 & $\mathrm{o}$ & M95 \\
\hline
\end{tabular}

Notes. $v_{2}$ : vibrational state; spin: ortho/para isomers.

References. C69 Cheung et al. (1969); M90 Menten et al. (1990); M91 Menten \& Melnick (1991); M95 Menten \& Young (1995).

\section{Appendix A: Water maser lines}

Table A.1 gives some properties of the sub-mm maser lines resolved by ALMA and the well-known $22 \mathrm{GHz}$ maser.

\section{Appendix B: Observations and data processing}

VY CMa was observed by ALMA on 2013 16-19 August using 16-20 $12 \mathrm{~m}$ antennas. The primary objective of these Science Verification (SV) observations was to demonstrate the ability to observe on baselines up to $2.7 \mathrm{~km}$ and develop calibration techniques involving strong, narrow spectral lines. Three separate configurations or scheduling blocks (SBs), covering each of the maser lines, are referred to as the $321 \mathrm{GHz}, 325 \mathrm{GHz}$, and $658 \mathrm{GHz}$ SBs; details are given in Table B.1. These were divided into one or more spectral windows (spw) covering $\sim 850-1700 \mathrm{~km} \mathrm{~s}^{-1}$ (depending on SB). Each spw was divided into 3840 channels, but as a result of Hanning smoothing in the correlator, the finest effective velocity resolution is approximately double the channel spacing, that is, $0.45 \mathrm{~km} \mathrm{~s}^{-1}$ for 321 and $658 \mathrm{GHz}$, and $0.9 \mathrm{~km} \mathrm{~s}^{-1}$ at $325 \mathrm{GHz}$. The data and scripts (including a description of the procedures) used for calibration and initial imaging are available from http://almascience. eso.org/alma-data/science-verification. As a Science Verification project, some observational methods were experimental, for example the duration of phase-referencing cycles turned out to allow a few phase ambiguities at the highest frequencies. Methods such as band-to-band phase transfer and fast switching will be available in future. These observations required very dry atmospheric conditions, so the weather determined their duration within the time available for Science Verification. Normal ALMA calibration and imaging procedures were followed, using CASA ${ }^{2}$. Each SB was executed three times at different hour angles, giving a total time on VY CMa at each frequency of $\sim 1.5 \mathrm{~h}$ in addition to calibration observations. The phase-reference source J0648-3044 was observed in $1.5 \mathrm{~min}$ scans, bracketing $6.75 \mathrm{~min}$ on VY CMa for the $321 \mathrm{GHz} \mathrm{SB}$ and $5.25 \mathrm{~min}$ for the 325 and $658 \mathrm{GHz}$ SBs.

Antenna positions were updated where required, and applied corrections derived from system temperature and water vapour radiometry measurements. The precipitable water vapour (PWV) was $0.3 \mathrm{~mm}$ except for the last of the three $658 \mathrm{GHz}$ observations, when it was $0.7 \mathrm{~mm}$. The water vapour radiometry corrections produced very significant improvements, especially for the $658 \mathrm{SB}$ taken at $0.7 \mathrm{~mm}$ PWV. A small amount of bad data were excised.

The bright QSO J0522-3627 was used for bandpass calibration. This was observed for the default duration of $5.25 \mathrm{~min}$ in each of the three executions of the $321 \mathrm{GHz}$ SB. However,

\footnotetext{
2 http://casa.nrao.edu
}

Table B.1. Observational parameters.

\begin{tabular}{lcccccc}
\hline \hline $\begin{array}{l}\text { SB } \\
(\mathrm{GHz})\end{array}$ & $\begin{array}{c}\text { spw centre } \\
(\mathrm{GHz})\end{array}$ & $\begin{array}{c}\text { spw width } \\
(\mathrm{GHz})\end{array}$ & $\begin{array}{c}\Delta_{\nu \text { cont }} \\
(\mathrm{GHz})\end{array}$ & $\begin{array}{c}\sigma_{\text {rms cont }} \\
(\mathrm{mJy})\end{array}$ & $\begin{array}{c}\delta v \\
(\mathrm{kHz})\end{array}$ & $\begin{array}{c}\sigma_{\text {rms1 }} \\
(\mathrm{mJy})\end{array}$ \\
\hline 321 & 321.18305 & 0.9375 & 1.74 & 0.31 & 244.141 & 2.5 \\
321 & 322.44385 & 0.9375 & & & 244.141 & 2.5 \\
321 & 310.95845 & 0.9375 & & & 244.141 & 1.6 \\
321 & 309.95865 & 0.9375 & & & 244.141 & 1.7 \\
325 & 325.10740 & 1.873 & 2.25 & 1.1 & 488.281 & 7.5 \\
325 & 321.96140 & 1.873 & & & 488.281 & 1.8 \\
658 & 657.82750 & 1.872 & 0.4 & 2.4 & 488.281 & 20 \\
\hline
\end{tabular}

Notes. $\Delta_{v \text { cont }}$ : effective continuum bandwidth for the whole SB, giving noise $\sigma_{\text {rms cont }} . \delta v$ : unaveraged channel spacing; $\sigma_{\text {rms } 1}$ : noise in $1 \mathrm{~km} \mathrm{~s}^{-1}$ channels free from bright emission.

it was only observed for $2.5 \mathrm{~min}$ in each of the $325 \mathrm{GHz}$ and $658 \mathrm{GHz}$ SBs. After all calibration was complete, we checked the variation of the imaged continuum emission with frequency within each SB. The channel-to-channel position scatter was as expected from the signal-to-noise ratio $(\mathrm{S} / \mathrm{N})$, without any systematic position shift, and the flux density was consistent with the expected spectral index $\sim 2$, so we are satisfied that the bandpass does not lead to misleading results. The main symptom was that the noise rms decreased more shallowly than the expected inverse square-root dependence on the number of channels averaged. The position uncertainties were also affected by dynamic range limitations in imaging and by possibly incomplete modelling of the atmosphere in the deep water-absorption lines.

Pallas was used as the primary flux scale calibrator (ButlerJPL-Horizons 2012, ALMA Memo 594), selecting baselines shorter than the first null in the visibilities. Using the $321 \mathrm{GHz}$ $\mathrm{SB}$, the flux density derived from Pallas for the phase-reference source, J0648-3044, was $0.433 \pm 0.008 \mathrm{Jy}$ at reference frequency $316.093 \mathrm{GHz}$, spectral index $\alpha-0.80 \pm 0.03$. Since the $325 \mathrm{GHz}$ data covered similar frequencies but had a poorer $\mathrm{S} / \mathrm{N}$ than the $321 \mathrm{GHz}$ data, we extrapolated the $321 \mathrm{GHz}$ values to the relevant frequencies for the $325 \mathrm{GHz}$ data. At $658 \mathrm{GHz}$, the flux density of J0648-3044 derived from Pallas is $0.28 \mathrm{Jy}$, compared with $0.24 \pm 0.02 \mathrm{Jy}$ extrapolated from $321 \mathrm{GHz}$. This may not be a fair comparison, since there is no guarantee that the spectral index is linear from 321 to $658 \mathrm{GHz}$, but it suggests that the error could be up to $15 \%$.

The phase-reference source, J0648-3044, $9^{\circ}$ from VY CMa, was used to derive time-dependent phase and amplitude corrections. The phase could be connected smoothly between successive scans for most antennas and times, but in a few cases where there was an ambiguity, the target scan affected was excluded from the initial imaging.

After applying instrumental and calibration source corrections, the VY CMa data in each SB was adjusted to constant velocity with respect to the local standard of rest (LSR). All velocities are given as $V_{\mathrm{LSR}}$. Low-resolution cubes were made for each data set to identify line-free continuum, and we made preliminary images to check the astrometry. In each SB, the brightest maser channel was identified and imaged, providing a starting model for self-calibration. After several iterations, the solutions were applied to all channels. The solutions were applied to all channels and to the data initially excluded because of the phasereferencing ambiguities noted above.

The bandwidth corresponding to the sum of line-free channels (spread over the whole observing bandwidth) $\Delta_{v \text { cont }}$ and the image noise rms $\sigma_{\text {rms cont. }}$ are given in Table B.1. The mean frequencies were 316 and $319 \mathrm{GHz}$ for the data sets referred 
to as 321 and $325 \mathrm{GHz}$. The continuum channels were imaged using natural weighting, which gave a synthesised beam of $\left(0{ }^{\prime} 22 \times 00^{\prime} 13\right)$ at 321 and $325 \mathrm{GHz}$, and $\left(00^{\prime} 11 \times 00^{\prime} 06\right)$ at $658 \mathrm{GHz}$. In all cases the beam position angle (PA) was $\sim 28^{\circ}$.

The shortest baseline was $14 \mathrm{~m}$, and inspection of the visibility amplitudes against baseline length shows that the flux density remains quite steady out to $70 \mathrm{~m}$ at $658 \mathrm{GHz}$ and $170 \mathrm{~m}$ at 321-325 GHz, suggesting that we recover all the flux on scales $<6^{\prime \prime}$ or $<13^{\prime \prime}$ at the higher or lower frequencies. We compared the total continuum flux densities with literature values (Fu et al. 2012; Kamiński et al. 2013; Muller et al. 2007; Shinnaga et al. 2004). All the measurements using $\sim 1^{\prime \prime}$ aperture lie close to a spectral index of $2.2 \pm 0.2$. Those taken using a larger aperture, such as at $658 \mathrm{GHz}$ from Shinnaga et al. (2004) and by Knapp \& Woodhams (1993) using the James Clerk Maxwell Telescope (effective aparture $\sim 18^{\prime \prime}$ ), are higher, for instance $0.62 \pm 0.04 \mathrm{Jy}$ at $240 \mathrm{GHz}, 2.18 \pm 0.24 \mathrm{Jy}$ at $353 \mathrm{GHz}$ and $9.7 \pm 1.5 \mathrm{Jy}$ at $677 \mathrm{GHz}$. This suggests that there is an extended component of dust on scales larger than we sampled.

The continuum was subtracted from each data set and partial uniform weighting (Briggs weighting with robust $=0.5$ as defined by CASA) was used to image the masers, giving beam sizes of $\left(0{ }^{\prime} 18 \times 00^{\prime} 09\right)$ and $\left(00^{\prime} 088 \times 00^{\prime} 044\right)$ at $321 / 325$ and $658 \mathrm{GHz}$, respectively. No spectral averaging was applied, so the measurements for each maser channel are not completely independent as a result of the Hanning smoothing in the correlator. All image extents were $\leq 80 \%$ of the primary beam, so no primary beam correction was applied.

The maser peak brightnesses and $\mathrm{S} / \mathrm{N}$ in each of the cubes were $321 \mathrm{GHz}$ : $426.6 \mathrm{Jy}_{\mathrm{beam}^{-1}}$, S/N 2010; $325 \mathrm{GHz}$ : $271 \mathrm{Jy} \mathrm{beam}^{-1}$, S/N 1330; $658 \mathrm{GHz}$ : $361 \mathrm{Jybeam}^{-1}$, and S/N 764. In the maser line wings (not dynamic-range limited but affected by the atmosphere) the $\sigma_{\text {rms }}$ noise values were 4,15 , and $40 \mathrm{mJy}$ for 321,325 , and $658 \mathrm{GHz}$, respectively. The values for all spw at lower resolution are given in Table B.1.

We measured the positions of the masers and continuum peaks by fitting two-dimensional Gaussian components using the AIPS task SAD. We did not attempt to resolve the individual components since the smallest beam size $\sim 50$ mas is much larger than the probable maser beamed size, although this might be possible for the brightest masers. Thus all flux densities are measured over the restoring beam. The relative position uncertainties are given by (beam size)/S/N (for fairly sparse $u v$ coverage in narrow channels; Condon et al. 1998; Richards et al. 2012). We selected components $>3 \sigma_{\text {rms }}$ at 321 and $325 \mathrm{GHz}$ or $>4 \sigma_{\text {rms }}$ at $658 \mathrm{GHz}$ (where $\sigma_{\text {rms }}$ was measured separately offsource for each channel) and rejected those that obviously coincided with sidelobes. We rejected components that did not form series of at least three in successive channels, within the maximum position uncertainty.

The total flux in fitted maser components at 321, 325, and $658 \mathrm{GHz}$ is $95 \%, 65 \%$, and $76 \%$ of the integrated map flux density for each SB. However, the fraction of flux recovered in components is no higher for channels containing peaks $>100 \mathrm{Jy}$, implying that the main loss is due to deconvolution errors putting power into sidelobes, since the 325 and $658 \mathrm{GHz}$ masers are more affected by atmospheric conditions. The component selection method avoids locating spurious positions, at the expense of loss of peak flux. We grouped the maser components into features comprising series of components in successive channels within the position errors, as in Richards et al. (2012), and attempted to cross-match the clumps of different transitions but found no significant associations. In each case, $5-10 \%$ of features have pairs within 50 mas, $2 \mathrm{~km} \mathrm{~s}^{-1}$, but applying a 50 mas shift to one data set produces a similar number of pairs, so this seems like random coincidence.

\section{Appendix C: Maser modelling}

Figure 8 compares the physical conditions needed to excite the observed maser transitions (at $22 \mathrm{GHz}$ and 321 and $325 \mathrm{GHz}$ ) within the ground-vibrational state. Here, we present results obtained with an excitation model that included the combined effects of collisional excitation by $\mathrm{H}_{2}$, spontaneous radiative decay, and radiative trapping of infrared transitions. We adopted the latest quantal rate coefficients (Daniel et al. 2011) for collisionally induced transitions amongst the lowest 45 rotational states of ortho- and para- $\mathrm{H}_{2} \mathrm{O}$, together with an extrapolation (Neufeld 2010) to the next 75 rotational states. We treated the effects of radiative trapping with the use of an escape probability method and the assumption of a steep velocity gradient in a single direction (e.g. Neufeld \& Melnick 1991). The results plotted here are for an effective water column density, $N\left(\mathrm{H}_{2} \mathrm{O}\right)=n\left(\mathrm{H}_{2} \mathrm{O}\right) /(\mathrm{d} v / \mathrm{d} z)$ of $10^{17} \mathrm{~cm}^{-2}$ per $\mathrm{km} \mathrm{s}^{-1}$; based upon the density and velocity profiles obtained for VY CMa by Decin et al. (2006), this value is appropriate for the general outflow at distances in the range 150 to $750 \mathrm{au}$, corresponding to angular offsets of 125 to 625 mas (the region within which most of the maser spots are located). The plotted contours show, as a function of temperature and $\mathrm{H}_{2}$ density, the negative optical depths predicted in the direction of the velocity gradient. These optical depths were computed in the unsaturated limit, where the population inversion is assumed to be undiminished by the effects of stimulated emission.

The contours labelled zero mark the boundary of the region within which the level populations are inverted. That region covers a broad range of densities and temperatures for all three transitions considered, although the $321 \mathrm{GHz}$ maser gain drops rapidly below $\sim 1000 \mathrm{~K}$, as expected given its relatively high upper state energy. Similar calculations, not presented here, for the $658 \mathrm{GHz}$ transition show a similar behaviour; this transition, too, shows a significant maser gain only at high temperature. Clearly, in the limit of high density, the population inversion for any transition inevitably disappears as the level populations approach LTE. However, the quenching density above which the population inversion vanishes varies from transition to transition and is clearly lowest for the $325 \mathrm{GHz}$ transition. This behaviour may explain why the $325 \mathrm{GHz}$ spots, as plotted in Figure 5 (purple squares), have a larger inner boundary than the other masing transitions.

Despite the large overlap of the regions in parameter space within which strong maser amplification can occur (Fig. 8), there are few or no exact coincidences between the 321 and $325 \mathrm{GHz}$ maser spots observed simultaneously. As noted previously, this may simply reflect the tendency of the exponential amplification process to accentuate small differences in opacity. 